\title{
Effect of Curing Conditions of a Poly(4-vinylphenol) Gate Dielectric on the Performance of a Pentacene-based Thin Film Transistor
}

\author{
Minkyu Hwang, Hwa Sung Lee, Yunseok Jang, Jeong Ho Cho, Shichoon Lee, \\ Do Hwan Kim, and Kilwon Cho* \\ Department of Chemical Engineering / Polymer Research Institute, Pohang University of Science and Technology, \\ Pohang 790-784, Korea
}

Received October 21, 2008; Revised November 11, 2008; Accepted November 13, 2008

\begin{abstract}
We improved the performance of pentacene-based thin film transistors by changing the curing environment of poly(4-vinylphenol) (PVP) gate dielectrics, while keeping the dielectric constant the same. The field-effect mobility of the pentacene TFTs constructed using the vacuum cured PVP was higher than that of the device based on the Ar flow cured gate dielectric, possibly due to the higher crystalline perfection of the pentacene films. The present results demonstrated that the curing conditions used can markedly affect the surface energy of polymer gate dielectrics, thereby affecting the field-effect mobility of TFTs based on those dielectrics.
\end{abstract}

Keywords: poly(4-vinylphenol), pentacene, transistor, OTFTs, surface energy.

\section{Introduction}

Organic thin-film transistors (OTFTs) have received considerable attention in recent years, and device performances comparable to those of amorphous silicon transistors have been achieved. Many groups have reported field-effect mobilities greater than $1 \mathrm{~cm}^{2} / \mathrm{Vs}$ for OFETs in which pentacene is used as the active layer. ${ }^{1-4}$ The surface characteristics of the gate dielectric strongly influence the quality of the gate dielectric-semiconductor interface hence the device performance..$^{5-7}$ In the last decade, considerable effort has been devoted to the fabrication of OTFTs using organic gate dielectrics $^{8-12}$ for use as switching devices for flexible displays. Although great advances have been made in the improvement of OTFTs using polymer gate dielectrics, there has been relatively little research into the effects of the surface energy of polymer gate dielectrics on device performance. ${ }^{13}$ Furthermore the effect of processing condition of polymer gate dielectric on the OTFTs device performance has never been reported. For polymer gate dielectrics the surface characteristic is affected much by the processing conditions especially for the cured polymer gate dielectrics such as poly(4-vinylphenol)(PVP).

Halik and coworkers showed that pentacene TFTs fabricated using PVP as the polymer gate-dielectric layer had fieldeffect mobilities as high as $3 \mathrm{~cm}^{2} / \mathrm{Vs} .{ }^{14}$ Katama and coworkers examined the effect of modifying the $\mathrm{SiO}_{2} / \mathrm{Si}$ substrate by spin-coating with a polymer thin film; they used several

*Corresponding Author. E-mail: kwcho@postech.ac.kr kinds of polymer, including poly(vinylidene fluoride), polystyrene, poly(methyl methacrylate), and poly(vinyl alcohol), which exhibited different surface energies. ${ }^{15}$ However, varying the type of polymer coating also changed other physical properties of the gate dielectrics, such as the dielectric constant and surface polarity. In the case of organic gate dielectrics, therefore, it is not easy to separate the effect of the surface energy of the gate dielectric from other effects. Moreover, in the case of PVP, the surface properties of a gate dielectric coated with this polymer are sensitive to the curing conditions such as the cure environment and schedule. This means that for the same chemical composition of the PVP pre-polymer, curing agent, and solvent, PVP gate dielectrics with different surface properties can be generated by varying the curing conditions.

In the present study, to investigate the effect of the surface energy of an organic gate dielectric on the electric properties of TFTs, we used a single type of polymer gate dielectric, PVP, and controlled the surface energy by simply changing the curing environment (i.e. in vacuum or in Ar gas flow). This approach made it possible to change only the surface energy while keeping the other properties of the gate dielectrics, such as the dielectric constant, the same.

\section{Experimental}

The $M_{w}$ of the PVP was $20,000 \mathrm{~g} / \mathrm{mol}$, and poly(melamineco-formaldehyde) (PMF) was used as the cross-linking agent. PVP and PMF were dissolved in propylene glycol methyl ether acetate (PGMEA) (about $15 \mathrm{wt} \%$ ) in a $1: 1$ equivalent 
Table I. Properties of PVP Gate Dielectrics with Various Curing Environmental Condition and Device Properties

\begin{tabular}{|c|c|c|c|c|c|c|c|c|c|c|}
\hline \multirow{3}{*}{$\begin{array}{l}\text { Curing } \\
\text { Condition }\end{array}$} & \multicolumn{7}{|c|}{ Gate Dielectric } & \multicolumn{3}{|c|}{ Device } \\
\hline & Con & tact Angle $\left({ }^{\circ}\right)$ & \multirow{2}{*}{$\begin{array}{c}\gamma_{s}^{p a} \\
\left(\mathrm{~mJ} / \mathrm{m}^{2}\right)\end{array}$} & \multirow{2}{*}{$\begin{array}{c}\gamma_{s}^{d a} \\
\left(\mathrm{~mJ} / \mathrm{m}^{2}\right)\end{array}$} & \multirow{2}{*}{$\begin{array}{c}\gamma_{s}^{b} \\
\left(\mathrm{~mJ} / \mathrm{m}^{2}\right)\end{array}$} & \multirow{2}{*}{$\begin{array}{c}\text { RMS } \\
\text { Roughness } \\
(\AA)\end{array}$} & \multirow{2}{*}{$\begin{array}{l}\text { Dielectric } \\
\text { Constant }\end{array}$} & \multirow{2}{*}{$\begin{array}{l}\text { Field-Effect } \\
\text { Mobility } \\
\left(\mathrm{cm}^{2} / \mathrm{Vs}\right)\end{array}$} & \multirow{2}{*}{$\begin{array}{c}\text { On/Off } \\
\text { Current } \\
\text { Ratio }\end{array}$} & \multirow{2}{*}{$\begin{array}{l}\text { Threshold } \\
\text { Voltage } \\
\text { (V) }\end{array}$} \\
\hline & Water & Diiodomethane & & & & & & & & \\
\hline Vacuum & 88 & 42 & 1.5 & 37.1 & 38.6 & 3 & $4.3 \pm 0.10$ & 0.87 & $3.7 \times 10^{4}$ & -18 \\
\hline Ar Gas & 70 & 31 & 7.6 & 38.1 & 45.7 & 3 & $4.2 \pm 0.15$ & 0.59 & $1.5 \times 10^{4}$ & -20 \\
\hline
\end{tabular}

${ }^{a} \gamma_{s}^{p}$ and $\gamma_{s}^{d}$ are the polar and dispersion components of the surface energy, respectively.

${ }^{b} \gamma_{s}$ is the surface energy of cured-PVP gate dielectrics. $\gamma_{s}=\gamma_{s}^{p}+\gamma_{s}^{d}$.

molar ratio. All chemicals were purchased from Aldrich and used without further purification. The gate dielectric films were formed by spin coating the prepared solutions at 3,000 rpm onto heavily doped n-type $\mathrm{Si}$ wafers as gate electrodes, with subsequent curing for $2 \mathrm{~h}$ at $200^{\circ} \mathrm{C}$ in a vacuum oven or Ar flow chamber.

The RMS roughness measured using an atomic force microscope (AFM) and the surface energies of the polymer gate dielectrics are listed in Table I. The surface energies of the gate dielectric films were evaluated by measuring the contact angles of two test liquids, distilled water and diiodomethane. ${ }^{16}$ The dielectric constants $(k)$ of the PVP gate dielectrics were obtained from $\mathrm{C}-\mathrm{V}$ measurements ${ }^{17}$ (Agilent 4284A) and the thickness of the PVP gate dielectrics were determined using ellipsometry. To investigate the chemical bonding states of the cured PVP gate dielectric surface, X-ray photoemission spectra (XPS) of the Ar gas flow and vacuum cured PVP surface were obtained using the 2B1 and 4B1 beam lines at the Pohang Accelerator Laboratory, Korea; an incident photon energy of $640 \mathrm{eV}$ was used to obtain the $\mathrm{C} 1 \mathrm{~s}$ and $\mathrm{N}$ 1s core level spectra. The $500 \AA$ pentacene film was deposited on top of the insulator surface at a deposition rate of $0.2 \AA / \mathrm{sec}$ and a substrate temperature of $50^{\circ} \mathrm{C}$ using an organic molecular beam deposition system. The structure of the pentacene films on gate dielectrics were investigated using X-ray diffraction (XRD) and AFM.

\section{Results and Disscusion}

Table I shows the surface properties of the PVP dielectrics cured either in a vacuum or Ar gas flow. The surface energy of the PVP cured under Ar gas flow conditions is higher than that of the PVP cured in a vacuum oven. Especially, the dispersion component of gate dielectrics is very similar, whereas the polar component of surface energy for each gate dielectric is quite different. To investigate the effects of the curing condition on surface composition of gate dielectrics, we obtained angle resolved C $1 \mathrm{~s}$ and $\mathrm{N}$ 1s spectra. Figure 1 shows the take-off angle dependent atomic ratio of differently cured PVP surfaces. For N 1s, the N/C intensity ratio from Ar gas flow cured PVP gate dielectric film is higher than vacuum cured one for various take-off angles, which causes higher polar component in surface energy due

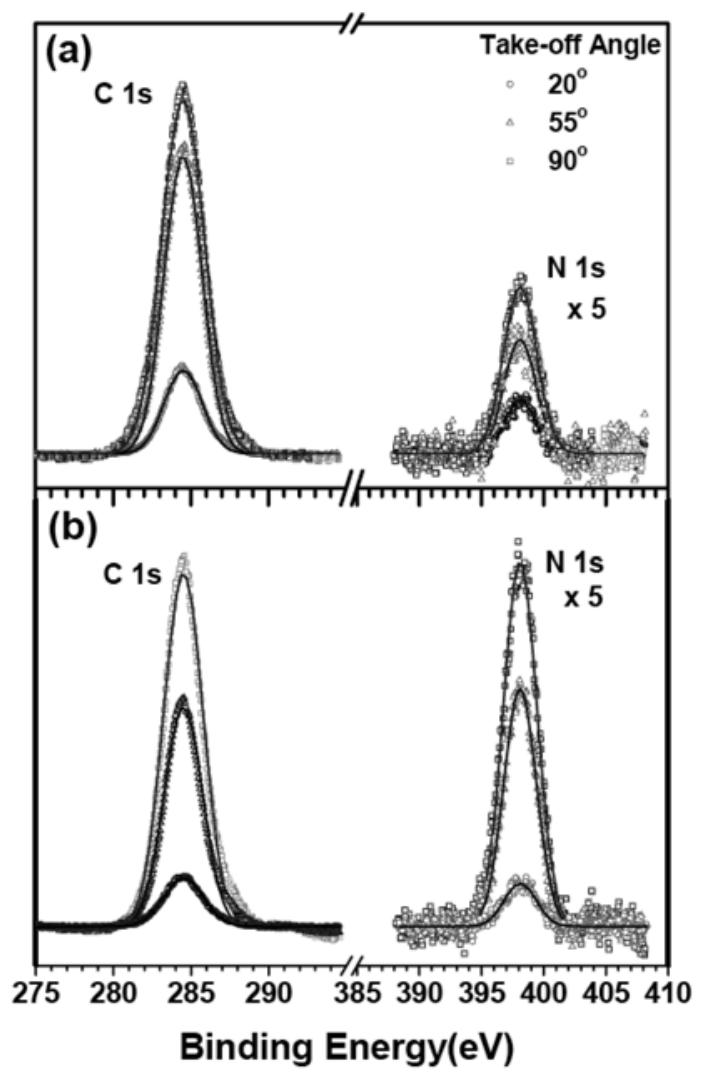

Figure 1. Angle-resolved high resolution $\mathrm{C} 1 \mathrm{~s}$ and $\mathrm{N}$ 1s X-ray photoemission spectra for a different gate dielectric: (a) vacuum cured PVP and (b) Ar gas flow cured PVP.

to the unshared electron pairs in $\mathrm{N}$ atoms. These results show that the cross-linking agents, PMF molecules are richer on the surface of Ar gas cured PVP gate dielectric than vacuum cured one because PMF molecules are more easily evaporated in vacuum condition than Ar gas flow condition.

Top-contact thin film transistors (TFTs) with a gold electrode (channel width $800 \mu \mathrm{m}$, length $100 \mu \mathrm{m}$ ) were used for analyzing the electrical characteristics of the pentacene TFTs. Current-voltage measurements were carried out in air at room temperature using Keithley 236 voltage source units. Figure 2(a) shows the drain current $\left(I_{D}\right)$ versus drain voltage $\left(V_{D}\right)$ curves obtained for the OTFTs fabricated using gate dielectrics with PVP films that were cured either under vac- 

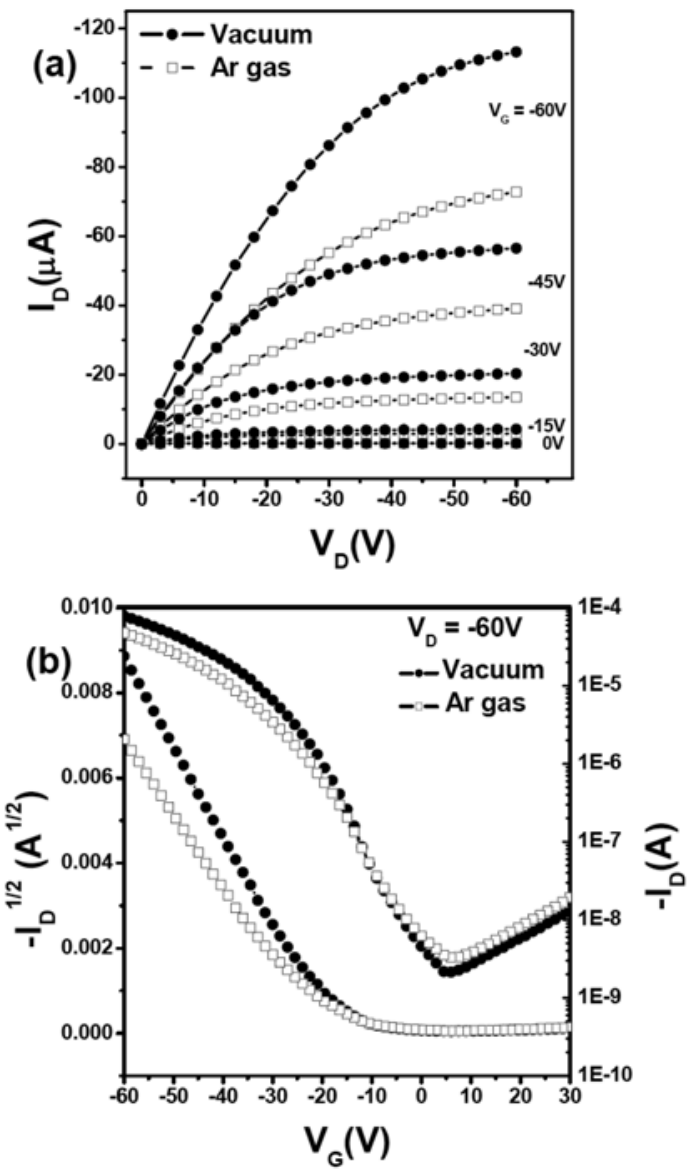

Figure 2. (a) Output curves and (b) transfer curves of pentacene TFTs with different gate dielectrics: (closed circles) vacuum cured PVP, (open squares) Ar gas flow cured PVP.

uum or Ar gas flow. These figures show typical output characteristics of transistors and saturation behavior at high drain voltage $\left(V_{D}\right)$. Interestingly, the maximum saturation current at each gate voltage was markedly higher for the gate dielectric with lower surface energy, i.e. vacuum cured PVP. Figure 2(b) shows plots of the square root of $\left|I_{D}\right|$ in the saturation region at $V_{D}=-60 \mathrm{~V}$ as a function of the gate voltage for the prepared devices. From each linear fit in Figure 2(b), the threshold voltage $\left(V_{T H}\right)$ and field-effect mobility $(\mu)$ in the saturation region were determined. ${ }^{17}$ The characteristic electric properties of the two devices are summarized in Table I. We found that the pentacene TFTs constructed using vacuum cured PVP showed higher average mobility $\left(0.87 \mathrm{~cm}^{2} / \mathrm{Vs}\right)$ than the corresponding TFTs fabricated using Ar gas flow cured PVP $\left(0.45 \mathrm{~cm}^{2} / \mathrm{Vs}\right)$. This tendency was always appeared in many experiment sets. The field-effect mobility of the pentacene TFTs thus increased as the surface energy of the gate dielectric decreased, in agreement with previous results. ${ }^{13}$ Surprisingly, our results show that a simple change in the curing condition of the gate dielectrics can have a marked effect on the field-effect mobility.
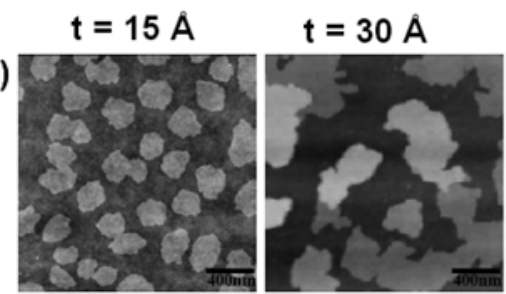

$t=500 A$

(b)
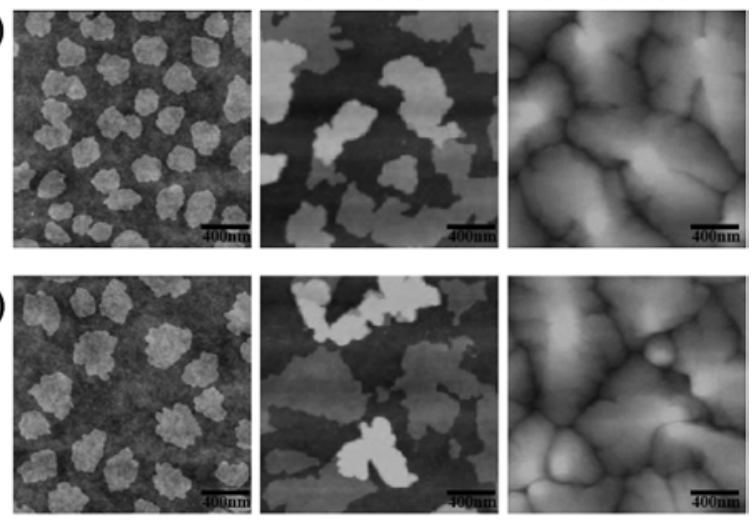

Figure 3. AFM images of pentacene films of three thicknesses on each gate dielectric: (a) pentacene on vacuum cured PVP and (b) pentacene on Ar gas flow cured PVP.

To determine the effect of the gate dielectric surface energy on the morphology and structure of the pentacene film, we performed AFM and XRD studies of the deposited pentacene film. Figure 3 shows AFM images of pentacene films with thicknesses of 15,30 and $500 \AA$ on each of the gate dielectrics. When the pentacene film thickness is $500 \AA$, the morphologies of the films on the two types of gate dielectric are very similar, with micron-sized pentacene crystalline grains being observed for both cases. For sub-monolayer coverage, however, larger crystalline grains were observed for the PVP surface cured under Ar gas flow (high surface energy) than for the PVP surface cured under vacuum. In general, larger crystalline grains of pentacene have a smaller number of grain boundaries, which results in a higher transport of carriers and hence a higher field-effect mobility. However, our results show that the TFTs with smaller pentacene crystalline grains has higher field-effect mobility, indicating that other aspects of these systems must be considered, such as crystal perfection and the interconnectivity of crystal grains.

Figure 4(a) shows the X-ray diffraction patterns for $50 \mathrm{~nm}$ thick pentacene layers on the two types of PVP gate dielectric. All peaks corresponding to $(00 l)$ planes come from the substrate-induced thin-film phase, which is characterized by an interplanar spacing of $15.4 \AA$ in the pentacene film. ${ }^{18}$ The bulk phase, which exhibits a vertical periodicity of $14.5 \AA$, was not observed in our results. The XRD patterns of the pentacene films on the two types of gate dielectric are quite similar. Next we examined the crystal perfection of the pentacene films on the cured PVP gate dielectrics. We determined the crystalline quality of each pentacene film from the rocking curve of the (002) reflection, ${ }^{19-22}$ as shown in Figure 4(b). The full-width at half-maximum (FWMH) value for the pentacene film on the vacuum cured PVP was 0.20 , whereas the FWMH value for the pentacene film on the Ar gas flow cured PVP was 0.29, indicating that the crystals formed on 

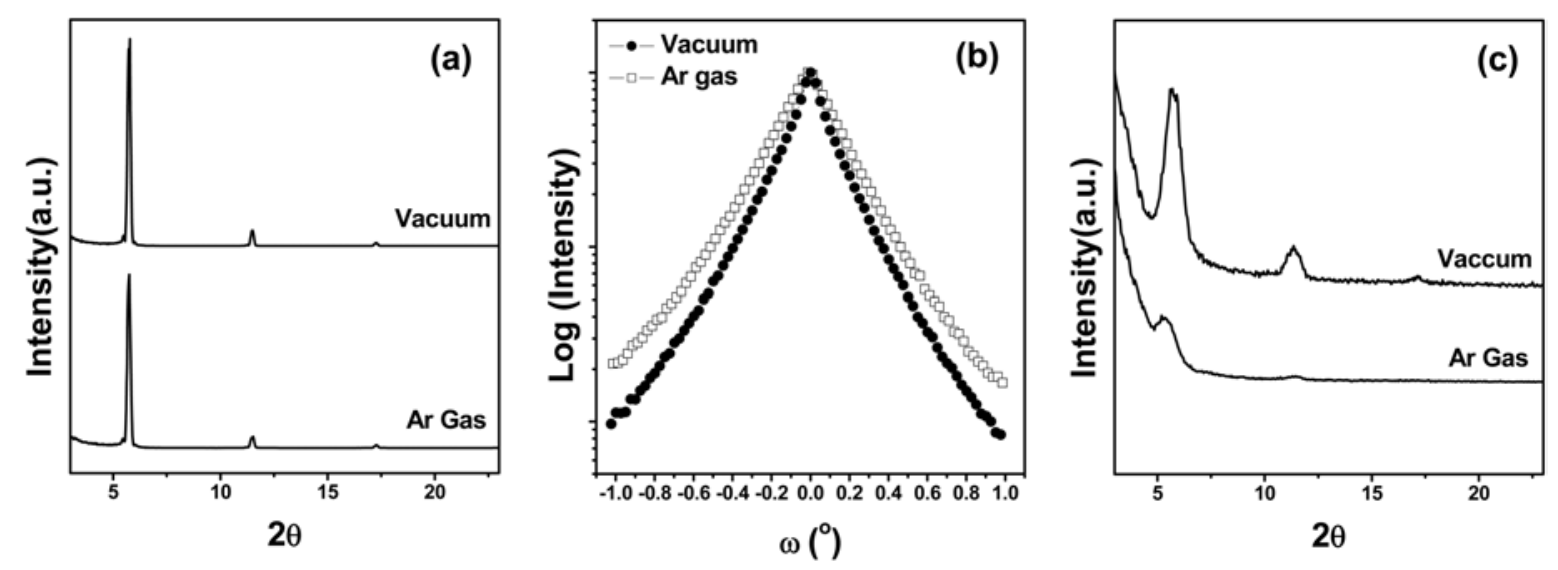

Figure 4. (a) X-ray diffraction patterns of $50 \mathrm{~nm}$ thickness pentacene films on vacuum cured PVP (top curve) and Ar gas flow cured PVP (bottom curve). (b) X-ray rocking curves of pentacene films on vacuum cured PVP (closed circles) and Ar gas flow cured PVP (open squares) at the center of the (002) Bragg reflections, and (c) X-ray diffraction patterns of $30 \AA$ thickness pentacene films on vacuum cured PVP (top curve) and Ar gas flow cured PVP (bottom curve).

the vacuum cured substrate have superior crystalline perfection. From these results we can infer that the surface energy of the gate dielectric influences the growth of and molecular arrangement within the pentacene film on the gate dielectric, which in turn affects the electrical properties of the final pentacene-based TFTs. We also performed XRD experiment for each thickness of pentacene film. $15 \AA$ thickness sample on PVP substrate was not shown the pentacene unique patterns. However, $30 \AA$ thickness pentacene samples were shown different patterns in Figure 4(c) XRD patterns are shown more well ordered pentacene film on vacuum cured PVP than pentacene on Ar gas cured PVP. This result was helpful to explain the surface energy of the gate dielectric influences the initial stage of pentacene nucleation and growth. The surface energy of a pentacene film was determined to be $42.1 \mathrm{~mJ} / \mathrm{m}^{2}$ by simple contact angle measurement. If the surface energy of the gate dielectric is higher than that of the pentacene film, pentacene molecules will more strongly bond to the surface of the gate dielectric than to the surrounding pentacene molecules, causing them to crystallize less perfectly. This would explain why of the two gate dielectrics tested, the Ar gas flow cured PVP gate dielectric, which has a higher surface energy, shows larger grains from the initial deposition stage (Figure 3 ) and a less perfect crystalline structure (Figure 4), which results in a lower value of the field-effect mobility. Taken together, our results suggest that the crystalline perfection is a more critical determinant of the carrier mobility than the initial grain size of the pentacene thin film on a PVP gate dielectric.

\section{Conclusions}

In summary, we improve the performance of pentacenebased thin film transistors by changing the curing environment (vacuum or Ar gas flow) of PVP gate dielectrics while keeping other parameters, such as the surface roughness and dielectric constant, the same. Relatively thick pentacene films of $500 \AA$ deposited on these gate dielectrics showed similar morphologies. For sub-monolayer pentacene coverages, however, the morphology and crystal perfection of the pentacene film was found to depend slightly on the surface energy of the PVP dielectric, which was determined by the curing environment used when fabricating the dielectric. The field-effect mobility of the pentacene TFTs constructed using a lower surface energy PVP cured under vacuum was higher than that of the corresponding TFTs constructed using Ar gas flow cured PVP.

Acknowledgments. This work was supported by a Korea Research Foundation Grant (KRF 2006-005-J01302). The authors are grateful to the Pohang Accelerator Laboratory for access to synchrotron radiation at the $2 \mathrm{~B} 1,4 \mathrm{~B} 1,3 \mathrm{C} 2$, and $8 \mathrm{C} 1$ beamlines.

\section{References}

(1) D. J. Gundlach, T. N. Jackson, D. G. Schlom, and S. F. Nelson, Appl. Phys. Lett., 74, 3302 (1999).

(2) H. S. Lee, D. H. Kim, J. H. Cho, M. Hwang, Y. Jang, and K. Cho, J. Am. Chem. Soc., 130, 10556 (2008).

(3) Y. Kato, S. Iba, R. Teramoto, T. Sekitani, T. Someya, H. Kawaguchi, and T. Sakurai, Appl. Phys. Lett., 84, 3789 (2004).

(4) D. Knipp, R. A. Street, A. Völkel, and J. Ho, J. Appl. Phys., 93, 347 (2003).

(5) C. D. Dimitrakopoulos and P. R. L. Malenfant, Adv. Mater., 14, 99 (2002).

(6) D. H. Kim, H. S. Lee, H. Yang, L. Yang, and K. Cho, Adv. Funct. Mater., 18, 1363 (2008).

(7) D. H. Kim, Y. D. Park, Y. Jang, H. Y. Yang, Y. H. Kim, J. I. Han, D. G. Moon, S. J. Park, T. H. Chang, C. H. Chang, M. K. Joo, C. Y. Ryu, and K. Cho, Adv. Func. Mater., 15, 77 (2005). 
(8) Y. Jang, D. H. Kim, Y. D. Park, J. H. Cho, M. Hwang, and K. Cho, Appl. Phys. Lett., 87, 152105 (2005).

(9) J. Veres, S. Ogier, and G. Lloyd, Chem. Mater., 16, 4543 (2004).

(10) N. Stutzmann, R. H. Friend, and H. Sirringhaus, Science, 299, 1881 (2003).

(11) A. Facchetti, M.-H. Yoon, and T. J. Marks, Adv. Mater., 17, 1705 (2005).

(12) H. E. Katz, Chem. Mater., 164748 (2004).

(13) S. Kobayashi, T. Nishikawa, T. Takenobu, S. Mori, T. Shimoda, T. Mitani, H. Shimotani, N. Yoshimoto, S. Ogawa, and Y. Iwasa, Nat. Mater., 3, 317 (2004).

(14) H. Klauk, M. Halik, U. Zschieschang, G. Schmid, W. Radlik, and W. Weber, J. Appl. Phys., 92, 5259 (2002).

(15) M. Yoshida, S. Uemura, T. Kodzasa, T. Kamata, M. Matsuzawa, and T. Kawai, Synth. Met., 137, 967 (2003).
(16) Y. Jang, J. H. Cho, D. H. Kim, Y. D. Park, M. Hwang, and K. Cho, Appl. Phys. Lett., 90, 132104 (2007).

(17) N. Arora, MOSFET Models for VLSI Circuit Simulation Theory and Practice, Springer-Verlag, New York, 1993, Ch. 4 \& 9.

(18) C. D. Dimitrakopoulos, A. R. Brown, and A. Pomp, J. Appl. Phys., 80, 2501 (1996).

(19) B. Nickel, R. Barabash, R. Ruiz, N. Koch, A. Kahn, L. C. Feldman, R. F. Haglund, and G. Scoles, Phys. Rev. B, 70, 125401 (2004).

(20) B. Kim, D. Kim, J. Chung, Y. J. Kim, I. Seo, S. K. Kwon, and K. Song, Polymer(Korea), 30, 362 (2006).

(21) J. Y. Ha, S. J. Yoon, D. Y. Jeong, and Y. Cho, Macromol. Res., 15, 86 (2007).

(22) H. S. Lee, D. H. Kim, J. H. Cho, Y. D. Park, J. S. Kim, and K. Cho, Adv. Funct. Mater., 16, 1859 (2006). 